\title{
Secondary Fluorescence Correction of 3D Heterogeneous Materials for Quantitative X-ray Microanalysis
}

Yu Yuan ${ }^{1}$, Hendrix Demers ${ }^{2}$, and Raynald Gauvin ${ }^{1}$

1. Department of Mining and Materials Engineering, McGill University, Montreal, Quebec, Canada.

2. Centre d'excellence en électrification des transports et stockage d'énergie, IREQ, Varennes, Québec, Canada.

MC X-Ray is a well-known Monte Carlo simulation program for qualitative and quantitative X-ray microanalysis [1]. It can simulate electron and $\mathrm{x}$-ray transport within sample using random numbers and physical models. Thus, full $x$-ray spectra and emitted x-ray intensity can be easily obtained. Also, it provides us full three-dimensional (3D) x-ray intensity distribution. However, the lack of secondary fluorescence limits the accuracy of MC X-Ray when dealing with samples with complicated geometry. The secondary fluorescence $\mathrm{x}$-rays are those emitted by $\mathrm{x}$-rays instead of electrons. It is normally ignored because of its small magnitude [2]. However, its contribution can be significant for samples with complicated geometry like multilayer materials, grain boundaries, and precipitates in alloy. The program of secondary fluorescence for bulk and multilayer materials has been developed using the 3D x-ray intensity distribution obtained from MC X-Ray as the input [3]. This work develops a new program to compute the secondary fluorescence emission for 3D heterogeneous materials. In this paper, the method and some results on sample with interface are shown.

In our program, the 3D heterogenous sample is simulated using voxels. Each voxel has its own chemical composition and three-dimensional coordinates, which represents its position. The primary characteristic and bremsstrahlung X-ray intensities (generated) for each voxel are obtained from MC X-Ray. Then, the secondary fluorescence $\mathrm{x}$-ray intensity, generated at certain voxel, emitted by $\mathrm{x}$-rays generated at other voxels are computed and summed up to acquire the total fluorescence x-ray intensity. Since x-rays travel much further than electrons, secondary fluorescence needs to be computed even for voxels where primary $\mathrm{x}$-ray intensity is zero. The computation is performed voxel by voxel until the contribution of certain voxel to the total fluorescence intensity is small enough. The voxel-based sample makes the program available for variety of sample geometries.

Take $\mathrm{Cu}$ and $\mathrm{Fe}$ with vertical interface as an example. As shown in Figure 3a) is the sample geometry for $\mathrm{Cu}$ and $\mathrm{Fe}$ with interface at $\mathrm{x}=0$. A series of $\mathrm{x}$-ray emission were simulated at various distances between interface and electron beam in the $\mathrm{Cu}$ region. The secondary fluorescence intensity for $\mathrm{Fe} \mathrm{K} \alpha$ is computed, which contributed from both characteristic fluorescence (emitted by $\mathrm{Cu} \mathrm{K \alpha}$ ) and bremsstrahlung fluorescence. Figure 1 shows the emitted $x$-ray intensity in three-dimensional distribution for primary intensity of $\mathrm{Cu} \mathrm{K} \alpha$ and characteristic fluorescence intensity of $\mathrm{Fe} \mathrm{K} \alpha$ when the distance between electron beam and interface of sample is $1 \mu \mathrm{m}$. The color of each point represents the $\mathrm{x}$-ray intensity. The comparison of Figure1 a) and b) shows that the secondary fluorescence has much larger interaction volume and much smaller magnitude than primary electron-induced $\mathrm{x}$-ray emission. Figure 2 shows the 2D x-ray intensity distribution for characteristic fluorescence of $\mathrm{Fe} \mathrm{Ka}$ in three planes, $\mathrm{x}=1.1 \mu \mathrm{m}$ (which gets close to the interface), $\mathrm{y}=0.1 \mu \mathrm{m}$, and $\mathrm{z}=0$. It is observed that the $\mathrm{Fe} \mathrm{K} \alpha$ can only be emitted at Fe Region (at $\mathrm{x}>1 \mu \mathrm{m}$ ) and the descending trend when the voxel position gets away from $\mathrm{Cu}$ region. Figure $3 \mathrm{~b}$ ) shows the variation of fluorescence intensity of $\mathrm{Fe} \mathrm{K} \alpha$ in the whole sample versus the distance between electron beam and sample interface. It is observed that the bremsstrahlung fluorescence intensity decreases as the distance increases since the bremsstrahlung $\mathrm{x}$ rays get away from Fe region as the electron beam moves. The characteristic fluorescence intensity increases first and then decreases. This increase of intensity when the distance changes from $0 \mu \mathrm{m}$ to 
$1 \mu \mathrm{m}$ is because the primary $\mathrm{Cu} \mathrm{K} \alpha$ intensity, as the source of characteristic fluorescence, increases to almost two times than before.

In the future, the accuracy of our program will be evaluated by comparing with experimental data.

References:

[1] R Gauvin and P Michaud, Microscopy and Microanalysis 15 (2009), p. 488-489.

[2] J Goldstein in "Scanning electron microscopy and x-ray microscopy", ed. D Writers, (Plenum Press, New York) p. 322.

[3] Y Yuan, H Demers, and R Gauvin, Microscopy and Microanalysis 23 (2017), p. 218-219.
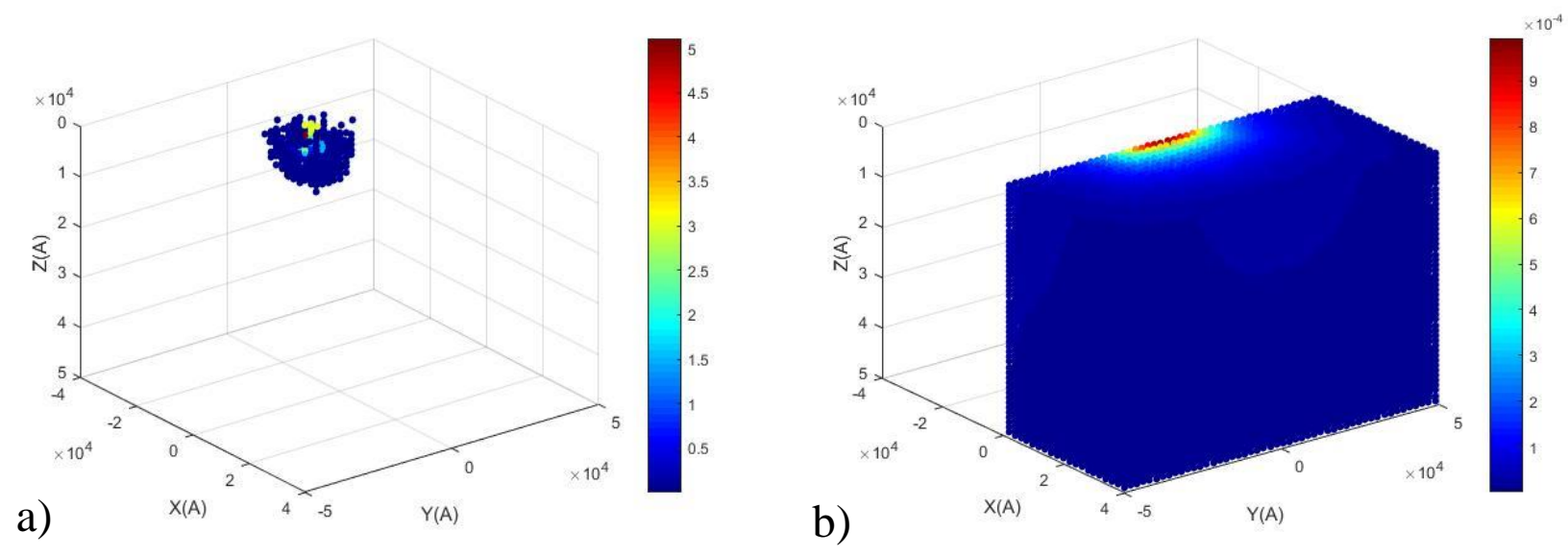

Figure 1. 3D x-ray intensity distribution for a) $\mathrm{Cu} K \alpha$ primary intensity and b) $\mathrm{Fe} \mathrm{K \alpha}$ characteristic fluorescence intensity emitted by $\mathrm{Cu} \mathrm{K} \alpha$ x-rays. The intensity is obtained for 100,000 electrons.

a)
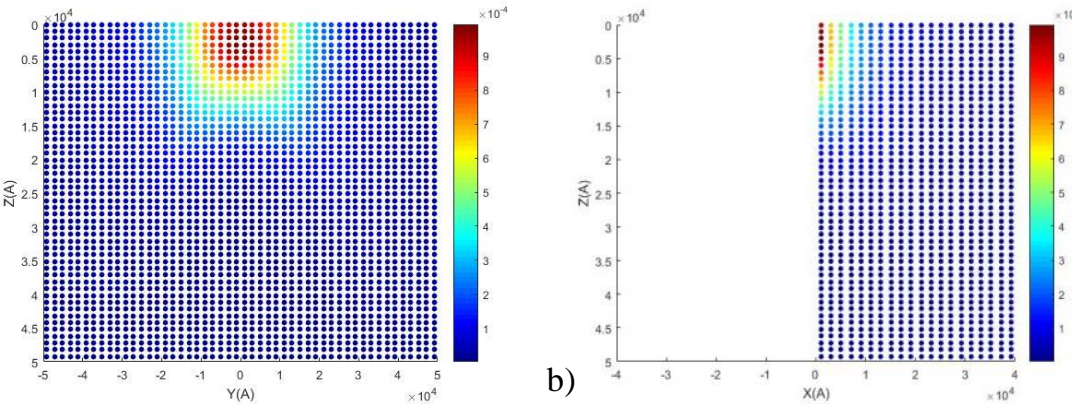

c)

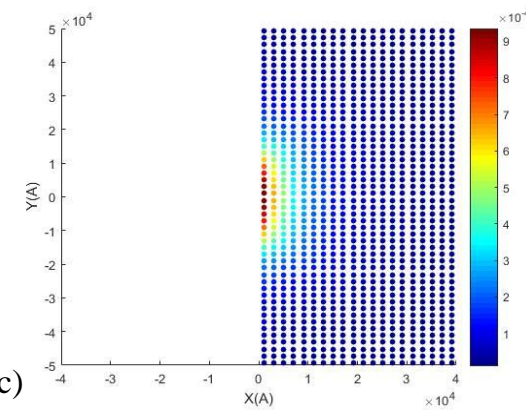

Figure 2. 2D x-ray intensity distribution for $\mathrm{Fe} \mathrm{K} \alpha$ fluorescence intensity emitted by $\mathrm{Cu} \mathrm{K} \alpha \mathrm{x}$-rays for plane a) $\mathrm{x}=1.1 \mu \mathrm{m}, \mathrm{b}) \mathrm{y}=0.1 \mu \mathrm{m}$, and c) $\mathrm{z}=0$. The intensity is obtained for 100,000 electrons.
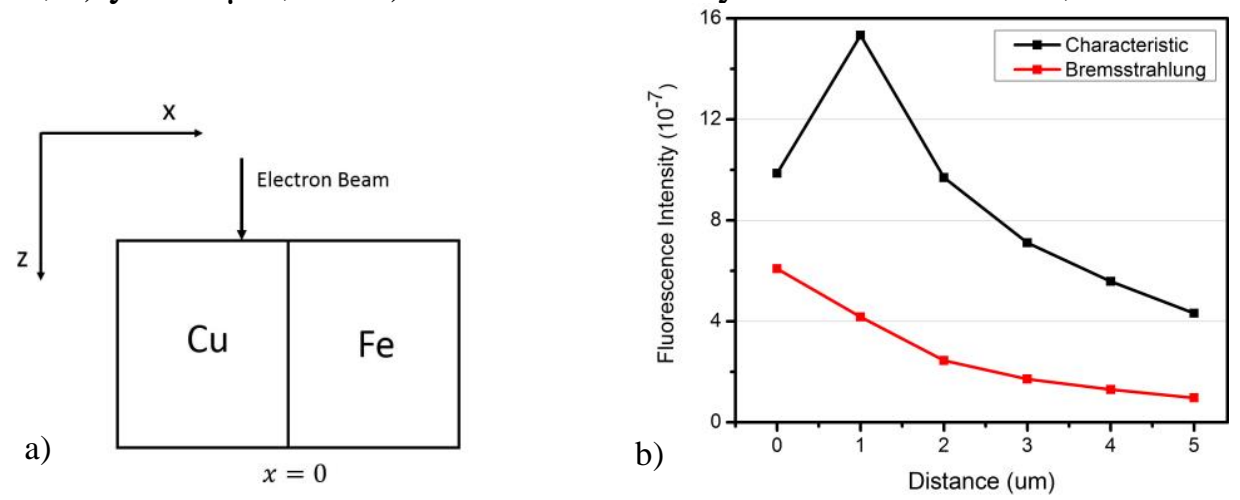

Figure 3. a) Sample geometry for $\mathrm{Cu}$ and Fe with interface at $\mathrm{x}=0$. b) The variation of secondary fluorescence intensity (per steradian per electron) of $\mathrm{Fe} \mathrm{K} \alpha$ versus the distance between interface and electron beam in the $\mathrm{Cu}$ region. 